\title{
Secure Transmission in IRS-Assisted MIMO Systems with Active Eavesdroppers
}

\author{
Ali Bereyhi*, Saba Asaad*, Ralf R. Müller*, Rafael F. Schaefer ${ }^{\dagger}$, and H. Vincent Poor ${ }^{\ddagger}$ \\ ${ }^{*}$ Institute for Digital Communications, Friedrich-Alexander Universität Erlangen-Nürnberg, Germany \\ ${ }^{\dagger}$ Information Theory and Applications Chair, Technische Universität Berlin, Germany \\ $\ddagger$ Department of Electrical Engineering, Princeton University, Princeton, NJ, USA \\ \{ali.bereyhi, saba.asaad, ralf.r.mueller\}@fau.de, rafael.schaefer@tu-berlin.de, poor@princton.edu
}

\begin{abstract}
This work studies secure transmission in intelligent reflecting surfaces (IRS)-assisted MIMO systems when an active eavesdropper is available in the network. We consider a scenario in which the eavesdropper performs an active pilot attack to contaminate the channel estimation at the base station. Invoking the method of secure regularized zero forcing, we develop an algorithm that designs beamforming vectors, as well as phaseshifts at the IRS, such that the active attacker is blinded. Our numerical investigations confirm that the proposed algorithm can suppress the active eavesdropper effectively, as long as legitimate and malicious terminals are statistically distinguishable.

Index Terms-Physical layer security, active pilot attack, intelligent reflecting surfaces, secure regularized zero forcing.
\end{abstract}

\section{INTRODUCTION}

Employing fixed intelligent metal sheets, known as intelligent reflecting surfaces (IRSs), has recently become a topic of significant interest in wireless communication; see for example [1]-[4]. The IRSs are composed of a large number of low cost units that receive signals from sources, customize them by basic operations, e.g., phase-shifts, and then forward the signal toward desired directions [5]. The use of IRSs boosts the spectral and energy efficiency of cellular networks without requiring power-hungry and expensive radio frequency chains [6]. All of these properties make IRSs a promising technology for new standards in wireless communication.

In this work, we study secure transmission in IRS-assisted multiple-input multiple-output (MIMO) systems. This topic has been widely investigated in the recent literature; see for example [7]-[11]. These lines of work usually consider a classic setting: An IRS-assisted MIMO transmitter transmits data to multiple user terminals (UTs) while some malicious receivers passively overhear the downlink channels. The ultimate goal is to jointly design the phase-shifts applied by the IRSs, and the precoding scheme at the transmitter, such that the achievable secrecy throughput is maximized. Considering standard models for IRSs, this objective presents a computationally intractable problem, due to the unit-modulus constraint imposed by the phase-shifts at IRSs. As the result,

This work was supported in part by the U.S. National Science Foundation under Grant CCF-1908308.

This work is accepted for presentation in the 2020 Asilomar Conference on Signals, Systems, and Computers. The link to the final version in the proceedings will be available later. various techniques, such as fractional programming, gradient projection, alternating optimization and Riemannian manifold optimization have been used to approximate the optimal beamformers and phase-shifts; see [12]-[15] for some instances of such approaches.

In this work, we deviate from the common lines of work and investigate the secrecy performance of IRS-assisted systems from a different viewpoint. Mainly, we study the capability of IRS-assisted MIMO systems in suppressing active malicious terminals. Our interest in this topic follows recent results on the so-called secrecy-for-free property of MIMO systems with large antenna arrays [16]-[18]. This property indicates that by standard beamforming toward legitimate UTs, passive eavesdroppers are blinded when antenna arrays grow large. In other words, the transmitter in this case does not need to take the presence of malicious terminals into account, since its narrow beamforming toward legitimate UTs implicitly suppresses eavesdroppers. This property is simply extended to IRS-assisted settings; see discussions in [18].

Unlike passive eavesdroppers, active attackers are not suppressed, unless their channel state information (CSI) is known by the transmitter [16]. This comes from the fact that active eavesdroppers contaminate the estimated CSI, and hence standard beamforming results in a non-vanishing leakage to the eavesdroppers; see [16], [19], [20] for more details. Following standard channel estimation techniques, it is further impractical to acquire CSI of eavesdroppers in the training phase 1 .

In this work, we consider the following question: Given the extra degrees of freedom achieved in IRS-assisted systems, is there a tractable approach by which active eavesdroppers are at least partially blinded? Our investigations give an interesting answer: Even by simple matched filtering at the transmitter, active eavesdroppers can still be suppressed when they are statistically distinguishable from legitimate UTs. The study further proposes a low-complexity algorithm for linear beamforming and phase-shift tuning at the IRS whose performance is investigated via numerical simulations.

\footnotetext{
${ }^{1}$ Nevertheless, existence of active attackers can detected via standard techniques; see for instance [21], [22].
} 


\section{A. Notation}

Throughout the manuscript, scalars, vectors, and matrices are indicated with non-bold, bold lower case, and bold upper case letters, respectively. $\mathbb{C}$ is the complex plane and the phase of $s$ is denoted by $\angle s$. The unit circle in the complex plane is shown by $\mathbb{U}$, i.e.,

$$
\mathbb{U}=\{z \in \mathbb{C}:|z|=1\} .
$$

$\mathbf{H}^{\top}$ and $\mathbf{H}^{\mathrm{H}}$ represent the transpose and transposed conjugate of $\mathbf{H}$, respectively. $\operatorname{tr}\{\mathbf{H}\}$ is the trace of $\mathbf{H}$. $\mathbb{E}\{\cdot\}$ denotes expectation. $[x]^{+}:=\max \{0, x\}$, and $[N]$ is used to represent $\{1, \ldots, N\}$. The notation $[N] \backslash n$ denotes $[N]$ with the integer $n$ being excluded from the set, i.e., $\{n\} \cup[N] \backslash n=[N]$.

\section{Problem Formulation}

Consider a multiuser MIMO setting. For sake of simplicity, we focus on a single-cell network in which $K$ single-antenna UTs are served by a base station (BS) with $N$ transmit antennas. To assist transmission, an IRS with $M$ antenna elements is further installed. The downlink signals are overheard by an active single-antenna eavesdropper which has access to the transmission codebooks and can perform active attacks in uplink transmission cycles.

The system is assumed to operate in time-division duplexing (TDD) mode. Hence, the uplink and downlink channels between a pair of transmitter and receiver are reciprocal. The BS estimates the CSI in the uplink training phase. It then employs its estimation to form the downlink transmit signal and radiates it toward receiving terminals. The radiated signal is also received via the IRS. Each antenna component at the IRS reflects its received copy of the signal toward UTs after applying a phase-shift on it.

A particular receiver observes a superposition of two major signal components: the one which is received through the direct path between the BS and the receiver, and the other being reflected via the IRS.

\section{A. System Model}

Let the BS transmit $\boldsymbol{x} \in \mathbb{C}^{N}$ in a given transmission time interval. The received signal at UT $k$ is given by

$$
y_{k}=y_{k}^{\mathrm{d}}+y_{k}^{\mathrm{r}}+z_{k}
$$

where $z_{k}$ models additive white Gaussian noise (AWGN) and reads $z_{k} \sim \mathcal{C N}\left(0, \sigma^{2}\right) . y_{k}^{\mathrm{d}}$ denotes the signal component received at UT $k$ through the direct path and reads

$$
y_{k}^{\mathrm{d}}=\mathbf{h}_{k}^{\top} \boldsymbol{x},
$$

with $\mathbf{h}_{k} \in \mathbb{C}^{N}$ being the uplink direct channel from UT $k$ to the BS. $y_{k}^{\mathrm{r}}$ further represents the reflected component and is given by

$$
\begin{aligned}
y_{k}^{\mathrm{r}} & =\mathbf{a}_{k}^{\top} \boldsymbol{\Theta} \mathbf{U}^{\top} \boldsymbol{x} \\
& =\boldsymbol{\theta}^{\top} \operatorname{diag}\left\{\mathbf{a}_{k}\right\} \mathbf{U}^{\top} \boldsymbol{x} \\
& =\boldsymbol{\theta}^{\top} \mathbf{F}_{k}^{\top} \boldsymbol{x}
\end{aligned}
$$

where $\mathbf{U} \in \mathbb{C}^{N \times M}$ and $\mathbf{a}_{k} \in \mathbb{C}^{M}$ denote the uplink channel from the IRS to the BS, and the uplink channel from UT $k$ to the IRS, respectively. Moreover, $\boldsymbol{\Theta}=\operatorname{diag}\{\boldsymbol{\theta}\}$ where $\boldsymbol{\theta}$ is an $M$-dimensional vector whose $m$-th entry is given by

$$
\theta_{m}=\vartheta_{m} \exp \left\{\mathrm{j} \phi_{m}\right\},
$$

with $\vartheta_{m} \in\{0,1\}$ modeling the activity of antenna element $m$, and $\phi_{m}$ being the phase-shift applied by the $m$-th element of the IRS. $\mathbf{F}_{k}$ is further defined as

$$
\mathbf{F}_{k}=\mathbf{U d i a g}\left\{\mathbf{a}_{k}\right\}
$$

and represents the effective uplink channel from UT $k$ to BS through the IRS.

Similarly, the received signal at the eavesdropper is given by

$$
y_{\mathrm{e}}=y_{\mathrm{e}}^{\mathrm{d}}+y_{\mathrm{e}}^{\mathrm{r}}+z_{\mathrm{e}}
$$

where $z_{\mathrm{e}} \sim \mathcal{C N}\left(0, \rho^{2}\right)$,

$$
y_{\mathrm{e}}^{\mathrm{d}}=\mathbf{h}_{\mathrm{e}}^{\top} \boldsymbol{x},
$$

with $\mathbf{h}_{\mathrm{e}} \in \mathbb{C}^{N}$ being the uplink channel from the eavesdropper to the BS, and

$$
\begin{aligned}
y_{\mathrm{e}}^{\mathrm{r}} & =\mathbf{a}_{\mathrm{e}}^{\top} \boldsymbol{\Theta} \mathbf{U}^{\top} \boldsymbol{x} \\
& =\boldsymbol{\theta}^{\top} \mathbf{F}_{\mathrm{e}}^{\top} \boldsymbol{x}
\end{aligned}
$$

for $\mathbf{a}_{\mathrm{e}} \in \mathbb{C}^{M}$ being the uplink channel from the eavesdropper to the IRS. As in (6), we further define the effective channel from the eavesdropper to the BS through the IRS as

$$
\mathbf{F}_{\mathrm{e}}=\mathbf{U} \operatorname{diag}\left\{\mathbf{a}_{\mathrm{e}}\right\} .
$$

For sake of brevity, in the remaining parts of the manuscript, we refer to the eavesdropper as UT e, wherever needed.

The vectors of channel coefficients model the path-loss, shadowing and small-scale fading effects. Depending on the environment, carrier frequency and topology of the network, the BS has a prior belief on the CSI which describes the fading process. This means that the BS knows first and second order statistics of the fading process. This is a typical assumption, since these parameters change very slowly in the system [23].

Remark 1: At this point, we do not restrict the analysis to a particular model and present the derivations for an arbitrary channel model. We later give explicit derivations for the conventional case of rich scattering environment in Section VI.

\section{ACQUiRIng CSI Under ACTIVE PILOT ATtack}

The channel estimation is performed in the uplink training phase. To this end, each UT transmits its own pilot of length $\tau$. We follow the recent class of channel estimation algorithms developed in [24], [25] for IRS-assisted systems.

Remark 2: Note that the focus of this study is on precoding and phase-shift design. We hence consider a basic channel estimation algorithm, and ignore the impact of noise, to keep the derivations tractable. The results are straightforwardly extended to other algorithms and the impact of noise can also be considered. 


\section{A. Pilot Structure and Channel Estimation Algorithm}

The pilots are assumed to be orthogonal and of the following structure: For UT $k \in[K]$, the pilot sequence $\psi_{k} \in \mathbb{C}^{\tau}$ is given by

$$
\boldsymbol{\psi}_{k}=\left[\begin{array}{c}
\boldsymbol{\mu}_{k} \\
\boldsymbol{\omega}_{k, 1} \\
\vdots \\
\boldsymbol{\omega}_{k, M}
\end{array}\right]
$$

where $\boldsymbol{\mu}_{k} \in \mathbb{C}^{\tau_{\mathrm{d}}}$ and $\boldsymbol{\omega}_{k, 1}, \ldots, \boldsymbol{\omega}_{k, M} \in \mathbb{C}^{\tau_{\mathrm{c}}}$ with $\tau_{\mathrm{d}}, \tau_{\mathrm{c}} \geq K$. $\boldsymbol{\mu}_{k}, \boldsymbol{\omega}_{k, 1}, \ldots, \boldsymbol{\omega}_{k, M}$ also construct orthogonal spaces for $k \in$ $[K]$, meaning that for $k \neq \ell$, we have

$$
\boldsymbol{\mu}_{k}^{\mathrm{H}} \boldsymbol{\mu}_{\ell}=0,
$$

and

$$
\boldsymbol{\omega}_{k, m}^{\mathrm{H}} \boldsymbol{\omega}_{\ell, m}=0,
$$

for $m \in[M]$. These pilots are assumed to be publicly known, meaning that the eavesdropper has also access to them.

The channel estimation is performed as follows:

1) In the first $\tau_{\mathrm{d}}$ symbol intervals, UT $k$ transmits $\boldsymbol{\mu}_{k}$ while the IRS is set off. The received signal is then used to estimate the coefficients of the direct channel.

2) Starting from symbol interval $\tau_{\mathrm{d}}+(m-1) \tau_{\mathrm{c}}+1$, user $k$ transmits $\boldsymbol{\omega}_{k, m}$ in $\tau_{\mathrm{c}}$ consequent symbol intervals while only the $m$-th element of the IRS is on, i.e., $\theta_{i}=0$ for $i \in[M] \backslash m$.

3) The BS estimates $\mathbf{f}_{k, m}$ by projecting the received signal onto the pilot sequences and canceling out the direct channel using its estimate from the first step.

\section{B. Active Pilot Attack}

Let $\ell$ denote the index of the legitimate UT that is overheard actively by the eavesdropper. To receive some information leakage, the eavesdropper transmits $\psi_{\ell}$. As the result, the received signals in the first $\tau_{\mathrm{d}}$ intervals can be written as

$$
\mathbf{Q}^{\mathrm{d}}=\sum_{k=1}^{K} \sqrt{P_{k}} \mathbf{h}_{k} \boldsymbol{\mu}_{k}^{\top}+\sqrt{P_{\mathrm{e}}} \mathbf{h}_{\mathrm{e}} \boldsymbol{\mu}_{\ell}^{\top}
$$

where $P_{k}$ and $P_{\mathrm{e}}$ denote the average transmit power of UT $k$ and eavesdropper, respectively.

Using $\mathbf{Q}^{\mathrm{d}}$, the BS estimates direct channel $\mathbf{h}_{k}$ as

$$
\hat{\mathbf{h}}_{k}=\frac{1}{\sqrt{P_{k}} \tau_{\mathrm{d}}} \mathbf{Q}^{\mathrm{d}} \boldsymbol{\mu}_{k}^{*}
$$

Following the orthogonality of the pilots, for $k \neq \ell$, we have $\hat{\mathbf{h}}_{k}=\mathbf{h}_{k}$. However, for UT $\ell$, we have

$$
\hat{\mathbf{h}}_{\ell}=\mathbf{h}_{\ell}+\sqrt{\frac{P_{\mathrm{e}}}{P_{\ell}}} \mathbf{h}_{\mathrm{e}} .
$$

In sub-frame $m$, i.e., between intervals $\tau_{\mathrm{d}}+(m-1) \tau_{\mathrm{c}}+1$ and $\tau_{\mathrm{d}}+m \tau_{\mathrm{c}}$, the eavesdropper transmits $\boldsymbol{\omega}_{\ell, m}$. Hence, the received signal at the BS in sub-frame $m$ is given by

$$
\begin{aligned}
\mathbf{Q}_{m}^{\mathrm{r}}=\sum_{k=1}^{K} & \sqrt{P_{k}}\left(\mathbf{h}_{k}+\mathbf{f}_{k, m}\right) \boldsymbol{\omega}_{k, m}^{\top} \\
& +\sqrt{P_{\mathrm{e}}}\left(\mathbf{h}_{\mathrm{e}}+\mathbf{f}_{\mathrm{e}, m}\right) \boldsymbol{\omega}_{\ell, m}^{\top}
\end{aligned}
$$

where $\mathbf{f}_{k, m}$ and $\mathbf{f}_{\mathrm{e}, m}$ represent the $m$-th column of $\mathbf{F}_{k}$ and $\mathbf{F}_{\mathrm{e}}$, respectively.

After projecting the received signal on the pilot sequences and canceling out the direct channel, the estimated effective channel is given by

$$
\hat{\mathbf{f}}_{k, m}=\frac{1}{\sqrt{P_{k}} \tau_{\mathrm{c}}} \mathbf{Q}_{m}^{\mathrm{r}} \boldsymbol{\omega}_{k, m}^{*}-\hat{\mathbf{h}}_{k} .
$$

For $k \neq \ell$, the estimated channel reads $\hat{\mathbf{f}}_{k, m}=\mathbf{f}_{k, m}$, and for the overheard user, it is given by

$$
\hat{\mathbf{f}}_{\ell, m}=\mathbf{f}_{\ell, m}+\sqrt{\frac{P_{\mathrm{e}}}{P_{\ell}}} \mathbf{f}_{\mathrm{e}, m} .
$$

Let us now define the end-to-end channel from UT $k$ with $k \in[K] \cup\{\mathrm{e}\}$ as

$$
\mathbf{g}_{k}(\boldsymbol{\theta})=\mathbf{h}_{k}+\mathbf{F}_{k} \boldsymbol{\theta} .
$$

By active pilot attack, we can conclude that for $k \in[K] \backslash\{\ell\}$, the transmitter has access to perfect CSI, i.e.,

$$
\hat{\mathbf{g}}_{k}(\boldsymbol{\theta})=\mathbf{g}_{k}(\boldsymbol{\theta}) .
$$

However, for $k=\ell$, the channel estimate is contaminated as

$$
\hat{\mathbf{g}}_{\ell}(\boldsymbol{\theta})=\mathbf{g}_{\ell}(\boldsymbol{\theta})+\sqrt{\alpha_{\mathrm{e}}} \mathbf{g}_{\mathrm{e}}(\boldsymbol{\theta})
$$

with $\alpha_{\mathrm{e}}=P_{\mathrm{e}} / P_{\ell}$. Note that we indicate $\boldsymbol{\theta}$ as an argument, as the end-to-end channel is modified by tuning the phase shifts at the IRS.

At the end of the uplink training phase, the BS encodes the estimated channel coefficients and transmits them to the UTs. Using an active attack detection algorithm, e.g., an energybased algorithm [21], the UTs detect existence of the active eavesdropper, and inform the BS over a feedback channel. The downlink CSI transmission and attack detection are performed in an interval of duration $\tau^{\mathrm{D}}$. Since error rates for these operations are significantly low, we further assume that they are error-free.

\section{Downlink Data Transmission}

Let $s_{k}$ denote the encoded information symbol of UT $k$. We assume that $s_{k} \sim \mathcal{C N}(0,1)$. The BS constructs its transmit signal $\boldsymbol{x}$ via linear precoding, meaning that

$$
\boldsymbol{x}=\sum_{k=1}^{K} s_{k} \mathbf{w}_{k} .
$$

Here, $\mathbf{w}_{k} \in \mathbb{C}^{N}$ is the beamforming vector of UT $k$ which is a function of estimated channel vectors, i.e., $\hat{\mathbf{h}}_{k}$ and $\hat{\mathbf{f}}_{k, m}$ for $k \in[K]$ and $m \in[M]$, and satisfies

$$
\left\|\mathbf{w}_{k}\right\|^{2}=P_{\mathrm{T}}
$$


for some per-user transmit power constraint $P_{\mathrm{T}}$. In this case, the received signal at $\mathrm{UT} k \in[K] \cup\{\mathrm{e}\}$ is given by

$$
y_{k}=\mathbf{g}_{k}^{\top}(\boldsymbol{\theta}) \mathbf{w}_{k} s_{k}+\sum_{j=1, j \neq k}^{K} \mathbf{g}_{k}^{\top}(\boldsymbol{\theta}) \mathbf{w}_{j} s_{j}+z_{k} .
$$

As it can be observed from (25), the received signal at UT $k$ is beamformed by both $\mathbf{w}_{k}$ and $\boldsymbol{\theta}$. Our main objective is hence to design the beamforming vectors and the phase-shifts, such that the eavesdropper is suppressed.

\section{A. Achievable Secrecy Sum-Rate}

To characterize the secrecy performance of the setting, we use the notion of ergodic secrecy rate: For UT $k$, a lower bound on the maximum achievable ergodic rate achieved in downlink data transmission phase is given by [26], [27]:

$$
R_{k}=\frac{T_{\mathrm{C}}-\tau-\tau^{\mathrm{D}}}{T_{\mathrm{C}}} \mathbb{E}\left\{\log \left(1+\mathrm{SINR}_{k}\right)\right\} .
$$

Here, $T_{\mathrm{C}}$ denotes duration of the coherence time interval2, and $\mathrm{SINR}_{k}$ is the signal to interference plus noise ratio (SINR) received at UT $k$ which is given by

$$
\mathrm{SINR}_{k}=\frac{\mathcal{S}_{k}}{\sigma^{2}+\mathcal{I}_{k}}
$$

where

$$
\begin{aligned}
\mathcal{S}_{k} & =\left|\mathbf{g}_{k}^{\top}(\boldsymbol{\theta}) \mathbf{w}_{k}\right|^{2}, \\
\mathcal{I}_{k} & =\sum_{j=1, j \neq k}^{K}\left|\mathbf{g}_{k}^{\top}(\boldsymbol{\theta}) \mathbf{w}_{j}\right|^{2} .
\end{aligned}
$$

Following [26], an achievable secrecy rate for UT $k \in[K]$ is given by subtracting the information leakage to the eavesdropper from $R_{k}$. Nevertheless, the exact characterization of information leakage in this setting is not a straightforward task to do. We hence follow a standard approach in which an upper bound on the information leakage is derived by considering a worst-case scenario: It is assumed that the eavesdropper is capable of acquiring its instantaneous CSI, as well as canceling the interference of other legitimate UTs. These assumptions lead to the following upper bound on the ergodic information leakage:

$$
R_{k}^{\mathrm{e}}=\frac{T_{\mathrm{C}}-\tau-\tau^{\mathrm{D}}}{T_{\mathrm{C}}} \mathbb{E}\left\{\log \left(1+\mathrm{ESNR}_{k}\right)\right\}
$$

where

$$
\mathrm{ESNR}_{k}=\frac{\left|\mathbf{g}_{\mathrm{e}}^{\top}(\boldsymbol{\theta}) \mathbf{w}_{k}\right|^{2}}{\rho^{2}}
$$

$R_{k}^{\mathrm{e}}$ characterizes the information rate leaked to the eavesdropper about encoded data of UT $k$. Note that for $k=\ell$, this rate is enhanced due to the active pilot attack. For $k \neq \ell$, this rate quantifies the leakage achieved by the eavesdropper via passive overhearing.

\footnotetext{
${ }^{2}$ In general, the coherence time interval of the direct and reflection paths could be different. For such cases, one could replace $T_{\mathrm{C}}$ with the minumum coherence time interval in the system.
}

From (26) and (30), a lower bound on the maximum achievable ergodic secrecy rate to UT $k$ is given by

$$
\begin{aligned}
R_{k}^{\mathrm{sec}} & =\left[R_{k}-R_{k}^{\mathrm{e}}\right]^{+} \\
& =\frac{T_{\mathrm{C}}-\tau-\tau^{\mathrm{D}}}{T_{\mathrm{C}}}\left[\mathbb{E}\left\{\log \frac{1+\mathrm{SINR}_{k}}{1+\mathrm{ESNR}_{k}}\right\}\right]^{+} .
\end{aligned}
$$

Note that the secrecy rate to UT $k$ achieved by this system is generally larger than the one given by $32 \mathrm{~b}$ ). The bound is however a good metric for performance characterization.

Using $R_{k}^{\mathrm{sec}}$, we define the achievable weighted secrecy sumrate $\bar{R}^{\text {sec }}$ as follows:

$$
\bar{R}^{\mathrm{sec}}=\sum_{k=1}^{K} \omega_{k} R_{k}^{\mathrm{sec}}
$$

for some wights $\omega_{1}, \ldots, \omega_{K}$ which model the priority of UTs in the network.

\section{Precoding and Phase-Tuning}

The optimal choice for the beamformers and phase-shifts are given via an optimization problem in which the weighted secrecy sum-rate $\bar{R}^{\text {sec }}$ is maximized over $\mathbf{w}_{1}, \ldots, \mathbf{w}_{K}$ and $\boldsymbol{\theta}$. Such an optimization however reduces to a non-deterministic polynomial-time (NP)-hard problem, and hence is not feasible to address in practice. We hence propose an alternative design approach considering the following two restrictions:

- The BS desires to process the estimated CSI as simply as possible, e.g., applying simple matched filtering. This follows the fact that beamformers are updated once per coherence time interval, and hence high computational load results in long processing time.

- Although the BS knows of the existence of the active eavesdropper, it does not have access to its instantaneous CSI and only knows its statistics. It hence must suppress the eavesdropper blindly.

We address these two issues by designing a stochastic form of secure regularized zero forcing (SRZF) precoding, recently proposed in [28].

\section{A. MRT-based Beamformers}

To address the complexity constraint, let us consider maximum ratio transmission (MRT) precoding. Extension to other linear approaches is skipped here and left for future studies. MRT beamforming simply sets

$$
\mathbf{w}_{k}=\sqrt{Q_{k}} \hat{\mathbf{g}}_{k}^{*}(\boldsymbol{\theta})
$$

for some $Q_{k}$ satisfying the transmit power constraint. Such an approach is however inefficient when an active eavesdropper is available in the network. This follows the fact that the estimated CSI is contaminated by the eavesdropper. To take this issue further into account, we modify the standard MRT approach as illustrated in the sequel.

From the viewpoint of UT $k$, the received signal is the superposition of $M+1$ components: One that is received through the direct path, and $M$ components that are reflected by the $M$ elements on the IRS. MRT suggests to construct 
$\mathbf{w}_{k}$ proportional to the filters matched to these components. Under certain conditions, these matched filters are superposed optimally via the same weighting imposed by the channel. This is however not the case when channel estimates are contaminated. We hence let the beamformers be arbitrary expansions of linear filters matched to the estimates of individual paths. In other words, for UT $k$, we set

$$
\begin{aligned}
\mathbf{w}_{k} & =\sqrt{Q_{k}}\left(\hat{\mathbf{h}}_{k}^{*}+\sum_{m=1} c_{k, m} \hat{\mathbf{f}}_{k, m}^{*}\right) \\
& =\sqrt{Q_{k}} \hat{\mathbf{g}}_{k}^{*}\left(\mathbf{c}_{k}\right)
\end{aligned}
$$

for some power factor $Q_{k}$, where $\mathbf{c}_{k}=\left[c_{k, 1}, \ldots, c_{k, M}\right]^{\top}$.

Considering (35b), the design of $\mathbf{w}_{k}$ reduces to the problem of finding $\mathbf{c}_{k}$. One should note that unlike $\mathbf{w}_{k}, \mathbf{c}_{k}$ does not change every coherence time interval. Hence, its calculation does not impose significant computational load on the system.

\section{B. Stochastic SRZF Precoding}

Our ultimate goal at the precoder is to invert the channel of legitimate UTs while keeping the eavesdropper blind. Considering $y_{k}$ given in (25), this goal is interpreted as

$$
\begin{array}{ll}
\min _{\mathbf{w}_{1}, \ldots, \mathbf{w}_{K}, \boldsymbol{\theta}} & \sum_{k=1}^{K}\left|\mathbf{g}_{k}^{\top}(\boldsymbol{\theta}) \mathbf{w}_{k}-1\right| \\
\text { subject to } & \sum_{k=1}^{K}\left|\mathbf{g}_{\mathrm{e}}^{\top}(\boldsymbol{\theta}) \mathbf{w}_{k}\right| \leq \epsilon
\end{array}
$$

for some small $\epsilon>0$. The unconstrained optimization in 36 solves the channel inversion task, and the constraint restricts leakage to the eavesdropper.

When the instantaneous CSI is available, (36) leads to the SRZF precoding scheme 3 . Nevertheless, it cannot be directly solved when the CSI is partially missing. To address this issue, we replace the instantaneous objective function and constraint with their expected values while considering the beamformers to be as given in (35b). This results in

$$
\begin{array}{ll}
\min _{\mathbf{c}_{1}, \ldots, \mathbf{c}_{K}, \boldsymbol{\theta}} & \sum_{k=1}^{K}\left|\mathbb{E}\left\{\mathbf{g}_{k}^{\top}(\boldsymbol{\theta}) \hat{\mathbf{g}}_{k}^{*}\left(\mathbf{c}_{k}\right)\right\}-\zeta_{k}\right| \\
\text { subject to } & \sum_{k=1}^{K}\left|\mathbb{E}\left\{\mathbf{g}_{\mathrm{e}}^{\top}(\boldsymbol{\theta}) \hat{\mathbf{g}}_{k}^{*}\left(\mathbf{c}_{k}\right)\right\}\right| \leq \epsilon
\end{array}
$$

for some scalars $\zeta_{1}, \ldots, \zeta_{K}$. The constrained optimization in (37) can be presented as an unconstrained problem with regularized objective. Using the method of Lagrange multipliers, we finally conclude the design for coefficients $\mathbf{c}_{1}, \ldots, \mathbf{c}_{K}$, and the phase-shifts as

$$
\left(\mathbf{c}_{1}, \ldots, \mathbf{c}_{K}, \boldsymbol{\theta}\right)=\underset{\substack{\mathbf{x}_{1}, \ldots, \mathbf{x}_{K} \in \mathbb{C}^{M} \\ \mathbf{y} \in \mathbb{U}^{M}}}{\operatorname{argmin}} F_{\mu}\left(\mathbf{x}_{1}, \ldots, \mathbf{x}_{K}, \mathbf{y}\right)
$$

where $F_{\mu}\left(\mathbf{x}_{1}, \ldots, \mathbf{x}_{K}, \mathbf{y}\right)$ is shown in 37 given at the top of the next page for some regularizer $\mu \in \mathbb{R}^{+}$.

\footnotetext{
${ }^{3}$ The formulation in 36 has a slight difference which we illustrate later.
}

Remark 3: The original form of the SRZF scheme follows the regularized least-squares (RLS) formulation in which both the regularization term and objective function are given by quadratic terms. This leads to a closed form solution which is given in [28]. From the compressive sensing point of view, the leakage is further suppressed by replacing the $\ell_{2}$-norm with the $\ell_{1}$-norm. In fact, one could look at the SRZF scheme as minimizing sum of $K$ leakage terms, i.e., $\left|\mathbf{g}_{\mathrm{e}}^{\top}(\boldsymbol{\theta}) \mathbf{w}_{k}\right|$ for $k \in[K]$, for the given channel inversion criteria. In this case, using the $\ell_{1}$-norm instead of the $\ell_{2}$-norm, the design results in an sparser vector of leakages, meaning that individual leakage terms are zero for larger numbers of UTs.

\section{Special Case of Rich Scattering Environments}

We now derive the objective function for the classical scenario of propagation in rich scattering environments. To keep the derivations tractable, we consider cases with no line of sight (LOS) channels and leave more general models for the extended version of the work.

Using the standard Rayleigh model for the fading process, the channel coefficients in this case are given by

$$
\begin{aligned}
\mathbf{h}_{k} & =\sqrt{\beta_{k}} \mathbf{T}_{k}^{1 / 2} \mathbf{h}_{k}^{0} \\
\mathbf{U} & =\mathbf{T}_{\mathrm{irs}}^{1 / 2} \mathbf{U}^{0} \mathbf{R}_{\mathrm{irs}}^{1 / 2} \\
\mathbf{a}_{k} & =\sqrt{\xi_{k}} \mathbf{V}_{k}^{1 / 2} \mathbf{a}_{k}^{0}
\end{aligned}
$$

for $k \in[K] \cup\{\mathrm{e}\}$, where $\mathbf{h}_{k}^{0}, \mathbf{U}^{0}$, and $\mathbf{a}_{k}^{0}$ are random operators with zero-mean and unit-variance independent and identically distributed (i.i.d.) complex Gaussian entries. The other parameters in this model are as follows:

- $\beta_{k}$ and $\mathbf{T}_{k} \in \mathbb{C}^{N \times N}$ capture the large-scale effects, e.g., path-loss and shadowing, and spatial correlation at the BS over the direct path from UT $k$ to the BS, respectively.

- $\mathbf{T}_{\text {irs }} \in \mathbb{C}^{N \times N}$ models spatial correlation observed at the BS considering signals reflected by the IRS, and $\mathbf{R}_{\text {irs }} \in$ $\mathbb{C}^{M \times M}$ denotes receive antenna correlation over the path from the BS to the IRS.

- $\xi_{k}$ and $\mathbf{V}_{k} \in \mathbb{C}^{M \times M}$ represent large-scale effects and spatial correlation at the IRS considering the signal received from UT $k$.

As indicated in the system model, it is assumed that $\mathbf{T}, \mathbf{R}$, $\beta_{k}$ and $\zeta_{k}$ are known at the BS.

Using basic properties of Gaussian random matrices, the objective function for this case is derived as in (42) in terms of a function $E_{k}(\mathbf{X}, \mathbf{Y})$ which is defined in (41) at the top of the next page.

\section{ITERATIVE ALGORITHM}

The proposed scheme in (39) does not lead to a tractable program due to the following two issues:

1) The objective function is not convex.

2) The phase shifts are restricted by the unit-modulus constraint, i.e., $\left|\theta_{m}\right|=1$.

In this section, we develop an iterative algorithm to approximate the solution of 39] with tractable complexity. 


$$
F_{\mu}\left(\mathbf{x}_{1}, \ldots, \mathbf{x}_{K}, \mathbf{y}\right):=\sum_{k=1}^{K}\left|\mathbb{E}\left\{\mathbf{g}_{k}^{\top}(\mathbf{y}) \hat{\mathbf{g}}_{k}^{*}\left(\mathbf{x}_{k}\right)\right\}-\zeta_{k}\right|+\mu \sum_{k=1}^{K}\left|\mathbb{E}\left\{\mathbf{g}_{\mathrm{e}}^{\top}(\mathbf{y}) \hat{\mathbf{g}}_{k}^{*}\left(\mathbf{x}_{k}\right)\right\}\right|
$$

$$
E_{k}(\mathbf{X}, \mathbf{Y})=\beta_{k} \operatorname{tr}\left\{\mathbf{T}_{k}\right\}+\xi_{k} \operatorname{tr}\left\{\mathbf{T}_{\mathrm{irs}}\right\} \operatorname{tr}\left\{\mathbf{R}_{\mathrm{irs}} \mathbf{X} \mathbf{V}_{k} \mathbf{Y}^{\mathrm{H}}\right\}
$$

$$
F_{\mu}\left(\mathbf{x}_{1}, \ldots, \mathbf{x}_{K}, \mathbf{y}\right)=\sum_{k=1}^{K}\left|E_{k}\left(\operatorname{diag}\left\{\mathbf{x}_{k}\right\}, \operatorname{diag}\{\mathbf{y}\}\right)\right|+\mu\left|E_{\mathrm{e}}\left(\operatorname{diag}\left\{\mathbf{x}_{\ell}\right\}, \operatorname{diag}\{\mathbf{y}\}\right)\right|
$$

We start the derivations by noting for a fixed $\mathbf{y}=\mathbf{y}_{0}$, the objective function of (39) is convex in $\mathbf{x}_{1}, \ldots, \mathbf{x}_{K}$. This observation suggests to use the alternating optimization technique: Starting from a $\mathbf{y}$, we first find the marginally optimal $\mathbf{x}_{1}, \ldots$, $\mathbf{x}_{K}$ while treating $\mathbf{y}$ as a fixed variable. We then set $\mathbf{x}_{1}, \ldots$, $\mathbf{x}_{K}$ to the determined solutions and update $\mathbf{y}$ by solving the marginal optimization in terms of $\mathbf{y}$. The alternation between these two optimizations is repeated until it converges.

In order to address the intractability issue imposed by the unit-modulus constraint, we use a classic relaxation technique. To this end, we note that for fixed $\mathbf{x}_{1}, \ldots \mathbf{x}_{K}$, the marginal optimization in terms of $\mathbf{y}$ has a convex objective with the non-convex constraint $\left|\mathrm{y}_{m}\right|=1$ for $m \in[M]$. We hence approximate the solution by solving the optimization problem for the convex constraint $\left|y_{m}\right| \leq 1$, and then projecting the solution onto the unit circle.

The proposed algorithm is presented in Algorithm 1 In this algorithm, the set $\hat{\mathbb{U}}$ denotes the relaxed support, i.e.,

$$
\hat{\mathrm{U}}=\{z \in \mathbb{C}:|z| \leq 1\} .
$$

\section{NUMERICAL EXPERIMENTS}

We evaluate the performance of the proposed scheme via some numerical experiments. To this end, a scenario with a single legitimate UT, i.e., $K=1$, and an eavesdropper is considered. The BS is equipped with $N=8$ antennas. The eavesdropper performs an active pilot attack with power ratio $\alpha_{\mathrm{e}}=0.5$.

\section{A. Channel Model}

To model the spatial correlation, the exponential channel model is considered [29]. For $n, n^{\prime} \in[N]$, we set

$$
\left[\mathbf{T}_{k}\right]_{n, n^{\prime}}=\mathrm{t}_{k}^{n-n^{\prime}}
$$

with

$$
\mathrm{t}_{k}=\exp \left\{\mathrm{j} \frac{2 \pi}{\lambda} \mathrm{d}_{\mathrm{bs}} \sin \phi_{k}^{\mathrm{t}} \sin \theta_{k}^{\mathrm{t}}\right\}
$$

where $\phi_{k}^{\mathrm{t}}$ and $\theta_{k}^{\mathrm{t}}$ are the azimuth and elevation angle of the average direction of arrival at UT $k \in\{\ell, \mathrm{e}\}$ over the direct path, respectively, and $\mathrm{d}_{\mathrm{bs}}$ denotes the distance between two
Algorithm 1 Stochastic SRZF via Alternating Optimization

Initiate Set $t=0, \boldsymbol{\theta}$ to some initial value $\boldsymbol{\theta}^{0}$, and

$$
\left(\mathbf{c}_{1}^{0}, \ldots, \mathbf{c}_{K}^{0}\right) \underset{\mathbf{x}_{1}, \ldots, \mathbf{x}_{K} \in \mathbb{C}^{M}}{\operatorname{argmin}} F_{\mu}\left(\mathbf{x}_{1}, \ldots, \mathbf{x}_{K}, \boldsymbol{\theta}^{0}\right)
$$

Let $\mathbf{C}^{0}=\left[\mathbf{c}_{1}^{0}, \ldots, \mathbf{c}_{K}^{0}\right]$.

while $\left\|\boldsymbol{\theta}^{t+1}-\boldsymbol{\theta}^{t}\right\|^{2} \geq \epsilon_{\theta}$ and $\left\|\mathbf{C}^{t+1}-\mathbf{C}^{t}\right\|_{F}^{2} \geq \epsilon_{\mathrm{c}}$

- Find $\hat{\boldsymbol{\theta}}^{t+1}$ as

$$
\hat{\boldsymbol{\theta}}^{t+1}=\underset{\mathbf{y} \in \hat{\mathbb{U}}^{M}}{\operatorname{argmin}} F_{\mu}\left(\mathbf{c}_{1}^{t}, \ldots, \mathbf{c}_{K}^{t}, \mathbf{y}\right)
$$

$\checkmark$ Set

$$
\theta_{t+1}=\angle \hat{\theta}^{t+1}
$$

- Update $\mathbf{C}^{t+1}=\left[\mathbf{c}_{1}^{t+1}, \ldots, \mathbf{c}_{K}^{t+1}\right]$ with

$$
\left(\mathbf{c}_{1}^{t+1}, \ldots, \mathbf{c}_{K}^{t+1}\right) \underset{\mathbf{x}_{1}, \ldots, \mathbf{x}_{K} \in \mathbb{C}^{M}}{\operatorname{argmin}} F_{\mu}\left(\mathbf{x}_{1}, \ldots, \mathbf{x}_{K}, \boldsymbol{\theta}^{t+1}\right)
$$

- Let $t \leftarrow t+1$

end while

neighboring antennas at the BS. Similarly, for $n, n^{\prime} \in[N]$ and $m, m^{\prime} \in[M]$, we set

$$
\begin{aligned}
& {\left[\mathbf{T}_{\mathrm{irs}}\right]_{n, n^{\prime}}=\mathrm{t}_{\mathrm{irs}}^{n-n^{\prime}}} \\
& {\left[\mathbf{R}_{\mathrm{irs}}\right]_{n, n^{\prime}}=\mathrm{r}_{\mathrm{irs}}^{m-m^{\prime}}}
\end{aligned}
$$

with

$$
\begin{aligned}
& \mathrm{t}_{\mathrm{irs}}=\exp \left\{\mathrm{j} \frac{2 \pi}{\lambda} \mathrm{d}_{\mathrm{bs}} \sin \phi_{\mathrm{irs}}^{\mathrm{t}} \sin \theta_{\mathrm{irs}}^{\mathrm{t}}\right\} \\
& \mathrm{r}_{\mathrm{irs}}=\exp \left\{\mathrm{j} \frac{2 \pi}{\lambda} \mathrm{d}_{\mathrm{irs}} \sin \phi_{\mathrm{irs}}^{\mathrm{r}} \sin \theta_{\mathrm{irs}}^{\mathrm{r}}\right\}
\end{aligned}
$$

where $\left(\phi_{\mathrm{irs}}^{\mathrm{t}}, \theta_{\mathrm{irs}}^{\mathrm{t}}\right)$ and $\left(\phi_{\mathrm{irs}}^{\mathrm{r}}, \theta_{\mathrm{irs}}^{\mathrm{r}}\right)$ are the tuples of azimuth and elevation angles corresponding to the direction of arrival and direction of departure at the IRS, respectively. $d_{\text {irs }}$ moreover denotes the distance between two neighboring antennas at the IRS.

For the spatial correlation matrix $\mathbf{V}_{k}$, we further set

$$
\left[\mathbf{V}_{k}\right]_{m, m^{\prime}}=\mathrm{v}_{k}^{m-m^{\prime}}
$$


with

$$
\mathrm{v}_{k}=\exp \left\{\mathrm{j} \frac{2 \pi}{\lambda} \mathrm{d}_{\mathrm{irs}} \sin \phi_{k}^{\mathrm{v}} \sin \theta_{k}^{\mathrm{v}}\right\}
$$

where $\phi_{k}^{\mathrm{v}}$ and $\theta_{k}^{\mathrm{v}}$ are the azimuth and elevation angle of the average direction of arrival at UT $k \in \cup\{\ell, \mathrm{e}\}$ over the reflection path, respectively.

The large-scale fading parameters $\beta_{k}$ and $\xi_{k}$ are further set for $k \in\{\ell, \mathrm{e}\}$ according to

$$
\begin{aligned}
& \beta_{k}=\beta_{0}\left(\frac{D_{k}^{\mathrm{d}}}{D_{0}}\right)^{-\alpha_{\mathrm{d}}} \\
& \xi_{k}=\xi_{0}\left(\frac{D_{k}^{\mathrm{r}}}{D_{0}}\right)^{-\alpha_{\mathrm{r}}}
\end{aligned}
$$

where $D_{0}$ is a reference distance, $\beta_{0}$ and $\xi_{0}$ represent the path-loss of the reference distance, and $\alpha_{\mathrm{d}}$ and $\alpha_{\mathrm{r}}$ denote the path-loss exponents for the direct and reflecting paths. $D_{k}^{\mathrm{d}}$ and $D_{k}^{\mathrm{r}}$ are the overall distance from the BS to UT $k \in\{1, \mathrm{e}\}$ over the direct and reflecting paths, respectively.

\section{B. Precoding and Phase-Tuning}

We use the beamforming vector in (35b) for downlink transmission. The coefficients of the beamformer, i.e., $\mathbf{c}_{\ell}$, as well as the phase-shifts at the IRS are determined using Algorithm 1 It is worth noting that these parameters are determined once prior to numerical simulations from the channel statistics, and then used to average over multiple coherence time intervals. To satisfy the transmit power constraint, the beamforming vector is normalized to its $\ell_{2}$-norm, i.e.,

$$
\mathbf{w}_{\ell}=\sqrt{P_{\mathrm{T}}} \frac{\hat{\mathbf{g}}_{\ell}^{*}\left(\boldsymbol{c}_{\ell}\right)}{\left\|\hat{\mathbf{g}}_{\ell}\left(\boldsymbol{c}_{\ell}\right)\right\|} .
$$

In the simulations, the regularizer is set to $\mu=1$.

\section{Simulation Results}

The simulations are given for $D_{0}=1, \beta_{0}=-10 \mathrm{~dB}$, and $\xi_{0}=-13 \mathrm{~dB}$. We further set $\alpha_{\mathrm{d}}=3.6$ and $\alpha_{\mathrm{r}}=2.1$. The position of the legitimate UT is generated randomly, and the angles of arrival and departure are determined accordingly. At the precoder, we set $P_{\mathrm{T}}=1$ and $\mathrm{d}_{\mathrm{bs}}=\lambda / 4$ where $\lambda$ denotes the wave-length. The distance between neighboring antennas at the IRS is further set to $d_{\text {irs }}=\lambda / 2$. The noise variances at the legitimate UT and the eavesdropper are $\sigma^{2}=\rho^{2}=0.1$.

Fig. 1 shows the achievable ergodic secrecy rate against the size of the IRS, i.e., $M$. Here, the $y$-axis shows the achievable secrecy rate to the legitimate UT, i.e., $k=1$. We further plot the achievable secrecy rate for a benchmark scheme in which the phase-shifts at the IRS are generated randomly and the BS uses conventional MRT precoding over the endto-end channel. As the figure shows, the proposed scheme significantly outperforms the benchmark. This observation indicates that the proposed scheme effectively suppresses the active eavesdropper using its statistics.

As another experiment, we consider a similar setting and set the number of elements at the IRS to $M=64$. We then fix the position of the legitimate UT and move the eavesdropper in

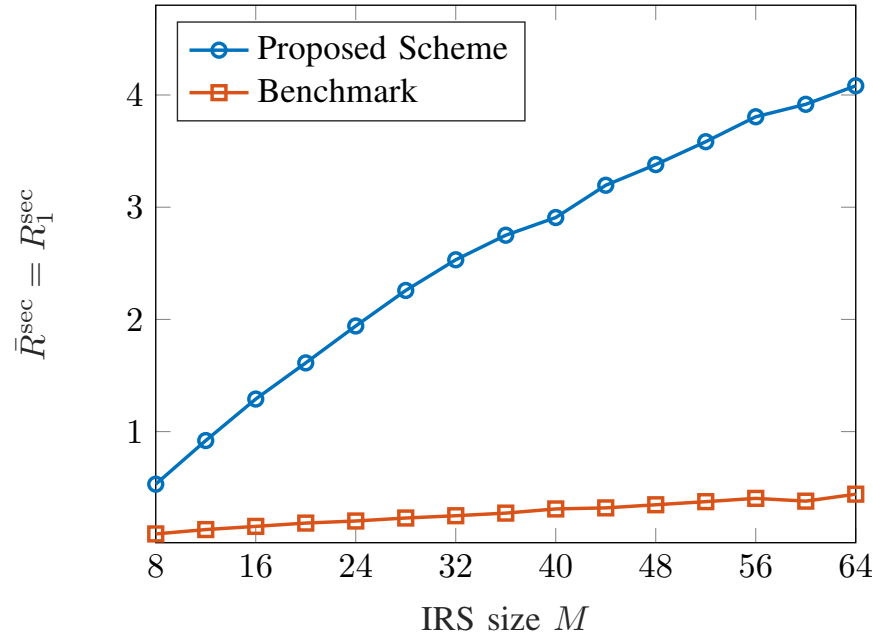

Fig. 1: Achievable secrecy rate vs. IRS size.

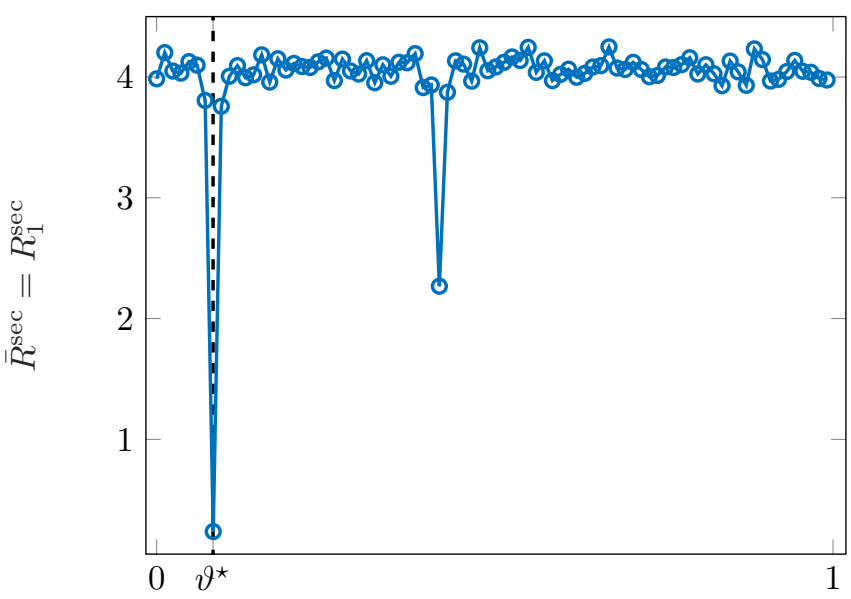

Sweeping parameter $\vartheta$

Fig. 2: Achievable secrecy rate vs. eavesdropper position.

a circle around the BS and IRS using a sweeping parameter $0 \leq \vartheta \leq 1$, such that at $\vartheta=\vartheta^{\star}$ the eavesdropper and the legitimate UT are seen at the same azimuth from the BS and IRS. The elevation of the UTs are also considerably close.

Fig. 2 shows the achievable secrecy rate against the sweeping factor. As it is observed, at $\vartheta=\vartheta^{\star}$, the achievable secrecy rate significantly drops. This observation comes from the fact that at this point, the spatial correlation matrices for the two terminals are almost the same. As the result, the BS cannot distinguish between these two receivers using their statistics.

\section{CONCLUSION}

We have studied the ability of IRS-assisted MIMO systems to suppress active eavesdroppers. In this respect, an iterative algorithm has been proposed that jointly performs precoding at the BS and tunes the phase-shifts at the IRS. Performance of the algorithm has been investigated through numerical experiments. 
The results of this study indicate that as long as the legitimate and malicious terminals are statistically distinguishable, eavesdroppers are significantly suppressed using the proposed technique for beamforming and phase-shift tuning. In addition to computational tractability, the algorithm enjoys low update rates, e.g., on the order of tens of coherence time intervals.

The analyses in this work have considered multiple idealistic assumptions, such as noise-free channel estimation, true prior belief on the channel model and accurate active attack detection. While these assumptions do not impact the final conclusion significantly, investigating the performance of the proposed algorithm under more realistic conditions is a natural direction for future work and is currently ongoing.

\section{REFERENCES}

[1] M. Di Renzo, M. Debbah, D.-T. Phan-Huy, A. Zappone, M.-S. Alouini, C. Yuen, V. Sciancalepore, G. C. Alexandropoulos, J. Hoydis, $\mathrm{H}$. Gacanin et al., "Smart radio environments empowered by reconfigurable AI meta-surfaces: An idea whose time has come," EURASIP Journal on Wireless Communications and Networking, vol. 2019, no. 1, pp. 1-20, May 2019.

[2] E. Basar, M. Di Renzo, J. De Rosny, M. Debbah, M.-S. Alouini, and R. Zhang, "Wireless communications through reconfigurable intelligent surfaces," IEEE Access, vol. 7, pp. 116753-116773, September 2019.

[3] Q. Wu and R. Zhang, "Intelligent reflecting surface enhanced wireless network via joint active and passive beamforming," IEEE Transactions on Wireless Communications, vol. 18, no. 11, pp. 5394-5409, November 2019.

[4] Q.-U.-A. Nadeem, A. Kammoun, A. Chaaban, M. Debbah, and M.-S. Alouini, "Intelligent reflecting surface assisted wireless communication: Modeling and channel estimation," arXiv preprint arXiv:1906.02360, December 2019.

[5] S. V. Hum and J. Perruisseau-Carrier, "Reconfigurable reflectarrays and array lenses for dynamic antenna beam control: A review," IEEE Transactions on Antennas and Propagation, vol. 62, no. 1, pp. 183-198, January 2014.

[6] C. Huang, A. Zappone, G. C. Alexandropoulos, M. Debbah, and C. Yuen, "Reconfigurable intelligent surfaces for energy efficiency in wireless communication," IEEE Transactions on Wireless Communications, vol. 18, no. 8, pp. 4157-4170, August 2019.

[7] B. Feng, Y. Wu, and M. Zheng, "Secure transmission strategy for intelligent reflecting surface enhanced wireless system," Proc. 11th International Conference on Wireless Communications and Signal Processing (WCSP), pp. 1-6, October 2019, China.

[8] D. Xu, X. Yu, Y. Sun, D. W. K. Ng, and R. Schober, "Resource allocation for secure IRS-assisted multiuser MISO systems," arXiv preprint arXiv:1907.03085, October 2019.

[9] X. Guan, Q. Wu, and R. Zhang, "Intelligent reflecting surface assisted secrecy communication via joint beamforming and jamming," arXiv preprint arXiv:1907.12839, December 2019.

[10] X. Yu, D. Xu, and R. Schober, "Enabling secure wireless communications via intelligent reflecting surfaces," arXiv preprint arXiv:1904.09573, February 2020.

[11] L. Dong and H. Wang, "Secure MIMO transmission via intelligent reflecting surface," IEEE Wireless Communications Letters, vol. 9, no. 6, pp. 787-790, January 2020 .

[12] X. Yu, J.-C. Shen, J. Zhang, and K. B. Letaief, "Alternating minimization algorithms for hybrid precoding in millimeter wave MIMO systems," IEEE Journal of Selected Topics in Signal Processing, vol. 10, no. 3, pp. 485-500, April 2016.

[13] H. Guo, Y.-C. Liang, J. Chen, and E. G. Larsson, "Weighted sumrate optimization for intelligent reflecting surface enhanced wireless networks," arXiv preprint arXiv:1905.07920, May 2019.

[14] A. Bereyhi, V. Jamali, R. R. Müller, A. M. Tulino, G. Fischer, and R. Schober, "A single-RF architecture for multiuser massive MIMO via reflecting surfaces," Proc. IEEE International Conference on Acoustics, Speech and Signal Processing (ICASSP), pp. 8688-8692, May 2020.
[15] S. Asaad, R. F. Schaefer, and H. Vincent Poor, "Hybrid precoding for secure transmission in reflect-array-assisted massive MIMO systems," Proc. IEEE International Conference on Acoustics, Speech and Signal Processing (ICASSP), pp. 8693-8697, May 2020.

[16] D. Kapetanovic, G. Zheng, and F. Rusek, "Physical layer security for massive MIMO: An overview on passive eavesdropping and active attacks," IEEE Communications Magazine, vol. 53, no. 6, pp. 21-27, June 2015.

[17] A. Bereyhi, S. Asaad, R. R. Müller, R. F. Schaefer, and A. M. Rabiei, "On robustness of massive MIMO systems against passive eavesdropping under antenna selection," Proc. IEEE Global Communications Conference (GLOBECOM), pp. 1-7, December 2018, Abu Dhabi, UAE.

[18] A. Bereyhi, S. Asaad, R. R. Müller, R. F. Schaefer, G. Fischer, and H. V. Poor, "Robustness of low-complexity massive MIMO architectures against passive eavesdropping," arXiv preprint arXiv:1912.02444, December 2019.

[19] S. Timilsina, G. A. A. Baduge, and R. F. Schaefer, "Secure communication in spectrum-sharing massive MIMO systems with active eavesdropping," IEEE Transactions on Cognitive Communications and Networking, vol. 4, no. 2, pp. 390-405, June 2018.

[20] D. Kudathanthirige, S. Timilsina, and G. A. A. Baduge, "Secure communication in relay-assisted massive MIMO downlink with active pilot attacks," IEEE Transactions on Information Forensics and Security, vol. 14, no. 11, pp. 2819-2833, November 2019.

[21] Q. Xiong, Y.-C. Liang, K. H. Li, and Y. Gong, "An energy-ratiobased approach for detecting pilot spoofing attack in multiple-antenna systems," IEEE Transactions on Information Forensics and Security, vol. 10, no. 5, pp. 932-940, May 2015.

[22] Q. Xiong, Y.-C. Liang, K. H. Li, Y. Gong, and S. Han, "Secure transmission against pilot spoofing attack: A two-way training-based scheme," IEEE Transactions on Information Forensics and Security, vol. 11, no. 5, pp. 1017-1026, May 2016.

[23] T. L. Marzetta, E. G. Larsson, H. Yang, and H. Q. Ngo, Fundamentals of Massive MIMO. Cambridge University Press, UK, 2016.

[24] Z. Wang, L. Liu, and S. Cui, "Channel estimation for intelligent reflecting surface assisted multiuser communications: Framework, algorithms, and analysis," arXiv preprint arXiv:1912.11783, April 2020.

[25] Z.-Q. He and X. Yuan, "Cascaded channel estimation for large intelligent metasurface assisted massive MIMO," IEEE Wireless Communications Letters, vol. 9, no. 2, pp. 210-214, February 2020.

[26] F. Oggier and B. Hassibi, "The secrecy capacity of the MIMO wiretap channel," IEEE Transactions on Information Theory, vol. 57, no. 8, pp. 4961-4972, 2011.

[27] R. F. Schaefer, G. Amarasuriya, and H. V. Poor, "Physical layer security in massive MIMO systems," Proc. Asilomar Conference on Signals, Systems, and Computers, pp. 3-8, December 2017, Pacific Grove, CA, USA.

[28] S. Asaad, A. Bereyhi, R. R. Müller, and R. F. Schaefer, "Secure regularized zero forcing for multiuser MIMOME channels," Proc. Asilomar Conference on Signals, Systems, and Computers, pp. 11081113, December 2019, Pacific Grove, CA, USA.

[29] S. L. Loyka, "Channel capacity of MIMO architecture using the exponential correlation matrix," IEEE Communications Letters, vol. 5, no. 9, pp. 369-371, September 2001. 